\title{
Two exchange-correlation functionals compared for first-principles liquid water
}

\author{
M. V. Fernández-Serra, ${ }^{1, *}$ G. Ferlat, ${ }^{2}$ and Emilio Artacho ${ }^{1}$ \\ ${ }^{1}$ Department of Earth Sciences, University of Cambridge, \\ Downing street, Cambridge CB2 3EQ, UK \\ ${ }^{2}$ Laboratoire de Minéralogie-Cristallographie, \\ Université Pierre et Marie Curie, Paris, France
}

(Dated: 27 July 2004)

\begin{abstract}
The first-principles description of liquid water using ab initio molecular dynamics (AIMD) based on Density Functional theory (DFT) has recently been found to require long equilibration times, giving too low diffusivities and a clear over-structuring of the liquid. In the light of these findings we compare here the room-temperature description offered by two different exchange correlation functionals: BLYP, the most popular for liquid water so far, and $\mathrm{RPBE}$, a revision of the widely used PBE. We find for RPBE a less structured liquid with radial distribution functions closer to the experimental ones than the ones of BLYP. The diffusivity obtained with RPBE for heavy water is still $20 \%$ lower than the corresponding experimental value, but it represents a substantial improvement on the BLYP value, one order of magnitude lower than experiment. These characteristics and the hydrogen-bond (HB) network imperfection point to an effective temperature $\sim 3 \%$ lower than the actual simulation temperature for the RPBE liquid, as compared with BLYP's $\sim 17 \%$ deviation. The too long $\mathrm{O}-\mathrm{O}$ average nearest-neighbor distance observed points to an excessively weak $\mathrm{HB}$, possibly compensating more fundamental errors in the DFT description.
\end{abstract}

\section{INTRODUCTION}

The interaction of organic pollutant molecules with the minerals and other components of the soil represents a paradigmatic case within the set of atomic-scale problems of relevance for the environment. It displays the level of complexity that demands a fundamental revision of the way we use computers for the simulation of condensed matter. On one hand the number of adsorption studies to address is enormous (combinations of many different pollutants, minerals, surfaces thereof etc.), on the other, each study displays a large degree of complexity, not least because of the wet character of these systems.

The effect of water in this and other wet systems is crucial in different ways, from the dielectric screening of electrostatic interactions to the active chemical role played in some important wet processes. These different aspects of water can be described at different levels of theory, including the static calculation of the system of interest immersed in an effective dielectric continuum, or the dynamic simulation of the liquid based on empirical potentials of different kinds. Even if simplified descriptions of water can be appropriate for some problems, other problems require the accuracy and transferability provided by first-principles simulation methods. This is not only our case, but it is true for many wet systems throughout geochemistry, biochemistry and wet chemistry. These considerations and the high variety of situations that we encounter in our pollutant problem (see Ref. 1 for the combinatorial aspects of the project and the workflow issues it entails) make it extremely important that we can use different simulation methods that involve different computational demands in a seamless fashion and within a single computational environment ${ }^{2.3 .4 .5}$ We need not only to interconnect the different simulations $\frac{6}{6}$ but that each one of them performs at the required level of accuracy.

On the first-principles side of the wet-systems description, ab initio molecular dynamics (AIMD) based on density-functional theory (DFT) is the technique that, on one hand, satisfies the needs mentioned above, and, on the other, is efficient enough to allow for the system sizes and time scales needed in the simulations with today's computers. Indeed, the last ten years have witnessed 
the success of many DFT-based AIMD simulations of liquid water, including work on structural, dynamical, chemical and electronic properties. $\frac{7,8,9,10,11,12,13}{4}$

Recent reports, $\stackrel{14.15 .16}{1}$ however, have questioned some of the results of earlier studies, showing that if the simulations are allowed to run for longer times, the diffusivity drops by one order of magnitude and the liquid becomes over-structured. The discrepancy with experiments 17.18 .19 is still unexplained. Prime suspects are the fundamental limitations of present-day AIMD simulations of liquid water: the inability of gradient-corrected (GGA) density functionals to describe dispersion interactions, and the neglect of quantum fluctuations in the classical description of the nuclear dynamics.

The need for long equilibration times and the poor structural and dynamical results have been further assessed in a recent contribution, ${ }^{20}$ where a relaxation process of a time scale larger than 20 ps was found at room temperature. It was observed to be related to rearrangements of the hydrogen-bond (HB) network in the liquid, expressed by the concentration of under-coordinated molecules, including a substantial presence (and relevance) of bi-coordinated ones. Both the radial distribution functions (RDFs) and the diffusivity were found to correlate strongly with the HB

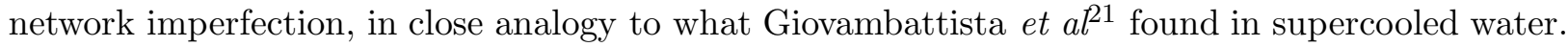
Furthermore, while the network was slowly evolving, the diffusivity was observed to equilibrate much faster to the instantaneous network state than to the final temperature. The mentioned paper also shows that the structure, diffusivity and network characteristics obtained, would very nicely fit experiment if they corresponded to an effective temperature of $\sim 240 \mathrm{~K}$, instead of the $300 \mathrm{~K}$ actually used in the AIMD simulations.

All those results were obtained for gradient-corrected (GGA) density functionals, specifically

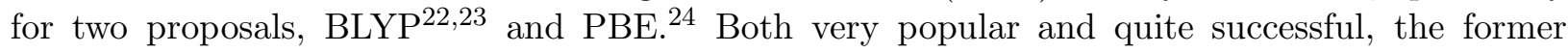
comes from the quantum-chemistry community and the latter from the condensed-matter physics community. Even if they were constructed with different criteria, from a fundamental point of view it is good news that both functionals give very similar results $\stackrel{14}{14}$ From a more practical view point, however, it would be highly desirable to have a satisfactory and efficient DFT description of liquid water in order to be able to address complex wet systems, especially since the more technical aspects for efficient DFT calculations (including linear scaling) have already been successfully tested for this system: 20

Alternative GGAs are being explored at the moment, and very promising results have been found by VandeVondele et al ${ }^{25}$, who tried several functionals finding much more satisfactory results. Since their functionals originate from the chemistry community, where special emphasis is put on accuracy for light elements, and since our interest in wet mineral surfaces involves heavier elements, we think it is worthwhile to complement their work with a similar study with functionals proposed more generally for the whole periodic table. In the following we present results for the structure, diffusivity and equilibration times for RPBE $\stackrel{26}{2}$ This GGA functional is a modification of PBE, well within its philosophy and fulfilling all its fundamental criteria. It is also very accessible from the points of view of coding (only a few lines differ from a PBE implementation) and computational demands, remaining at the GGA level. It has already been tested for varied systems, including systems with heavy elements. ${ }^{27,28}$ Functionals beyond GGA (meta-GGAs) $\stackrel{29.30}{2}$ hybrid functionals, $, 31.32,33$ and functionals including Van der Waals interactions 34,35 should also be checked, but their efficiency is lower, and the scope of wet systems that can be addressed at present is much narrower than for GGAs. None of these functionals will be addressed here.

Asthagiri et al $\underline{16}$ tested an alternative GGA, very similar to PBE as well, called revPBE $\underline{36}$ The spirit of the PBE modification of this functional is similar to RPBE (except for the fact that the latter respects the Lieb-Oxford bound locally, which ensures a global fulfilment of the condition for any electronic density) representing an alternative worth considering. Good revPBE results for liquid water were obtained by those authors 16 before the long time-scale relaxation problem was 
TABLE I: AIMD simulations performed in this work, all of them with periodic boundary conditions in a cubic cell of size $a=9.865 \AA$. DF stands for the particular density functional used, $T$ for the final equilibrated temperature, $\tau_{\text {sim }}$ for the AIMD simulation time after AIMD equilibration, $\tau_{\text {eq }}$ for the AIMD equilibration time, "Model" for the model used for preparation, $T_{\text {pre }}$ for the temperature at which the preparation model had been equilibrated and $T_{i}$ for the AIMD initial temperature (after the $\tau_{e q}$ anneal). Temperatures in $\mathrm{K}$ and times in ps.

\begin{tabular}{ccccclcc}
\hline \hline$\#$ & DF & $T$ & $\tau_{\text {sim }}$ & $\tau_{e q}$ & Model & $T_{\text {pre }}$ & $T_{i}$ \\
\hline 1 & BLYP & 298 & 20 & 4 & BLYP & 315 & 300 \\
2 & BLYP & 315 & 32 & 6 & SPC $/ E$ & 300 & 300 \\
3 & RPBE & 300 & 17 & 5 & BLYP & 315 & 300 \\
4 & BLYP & 345 & 30 & 4 & TIP5P & 325 & 325 \\
\hline \hline
\end{tabular}

reported. Under the new circumstances it seems that the equilibration and simulation times used there $\frac{16}{}$ could have been too short. A revision of those tests would also be useful, but is beyond the scope of the present work.

\section{METHOD}

The simulations were performed using the Kohn-Sham approach ${ }^{37}$ to DFT ${ }^{38}$ in the generalizedgradient approximation (GGA). The BLYP ${ }^{22.23}$ and $\mathrm{RPBE}^{26}$ exchange-correlation functionals were used and are compared in the following. Core electrons were replaced by norm-conserving pseudopotentials ${ }^{39}$ in their fully non-local representation ${ }^{40}$ Numerical atomic orbitals (NAO) of finite support were used as basis set, and the calculation of the self-consistent Hamiltonian and overlap matrices was done using the linear-scaling SIESTA method. ${ }^{41.42}$ Integrals beyond two-body were performed in a discretized real-space grid, its fineness determined by an energy cutoff of 150 Ry. A double- $\zeta$ polarized (DZP) NAO basis set was used, which had been obtained following the method proposed in Refs. 43 , 44 for a confining pressure ${ }^{44}$ of $0.2 \mathrm{GPa}$. The validation of the method, pseudopotentials and basis set can be found in Ref. 20, including the approximations required for linear scaling.

We performed AIMD simulations for 32 molecules of heavy water for the BLYP and RPBE density functionals (a detailed comparison between BLYP and PBE for this system can be found in Ref. 14). Details are given in Table【 AIMD equilibration is achieved by means of temperature annealing (velocity re-scaling) $\stackrel{45}{\stackrel{4}{w}}$ while the actual simulations are performed by Verlet's integration ${ }^{45}$ In all the simulations the time step used was $0.5 \mathrm{fs}$. The observed total-energy drifts corresponded to drifts in the system temperature between $0.26 \mathrm{~K} / \mathrm{ps}$ and $0.36 \mathrm{~K} / \mathrm{ps}$. The simulations were performed at constant volume, i.e., for fixed cell size and shape, under periodic boundary conditions. As in Ref. 20, the time scale of the HB relaxation process is monitored by following its non-equilibrium behaviour. The "moving time window" used in Ref. 20 is used to obtain the time dependence of the variables of interest.

Empirical simulations were performed using different force fields (TIP5P ${ }^{46}$ and SPC/E $\mathrm{E}^{47}$ ) in order to prepare reasonably equilibrated starting points for AIMD. These simulations were performed with the GROMACS MD package $\stackrel{48.49}{ }$ under constant volume and temperature conditions using a Berendsen-type thermostat. ${ }^{50}$ The empirical simulations were equilibrated during 200 ps. 

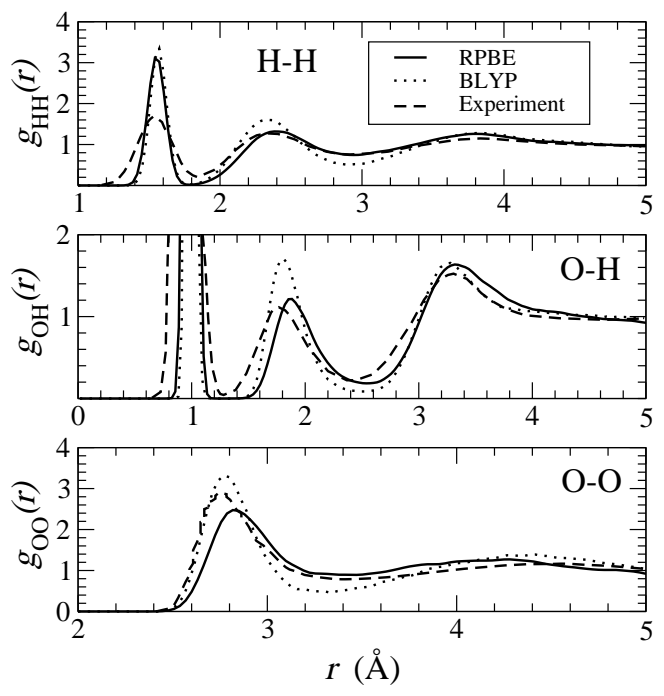

FIG. 1: Comparison of the $\mathrm{H}-\mathrm{H}, \mathrm{O}-\mathrm{H}$, and $\mathrm{O}-\mathrm{O}$ radial distribution functions as obtained in this work for 32 molecules with RPBE (solid line), with BLYP (dotted line) and by experiment ${ }^{18.19}$ (dashed line), at a temperature of $300 \mathrm{~K}$.

\section{RESULTS AND DISCUSSION}

Fig. 1 compares the RDFs obtained with RPBE and BLYP to experiment. Looking mainly at the inter-molecular features, the RDFs for RPBE are considerably less structured than the ones for BLYP, even if still over-structured with respect to experiment. The comparison in Ref. 14 between PBE and BLYP gave much closer results. The too large distance for the first peak position of the $\mathrm{O}-\mathrm{O}$ RDF is worth noting for RPBE, however. It indicates too weak HBs that could account for the more fluid character of the RPBE liquid as compared with BLYP or PBE. The fact that this $\mathrm{O}-\mathrm{O}$ distance is longer than the experimental value (possibly weaker HB) but the liquid remains still slightly overstructured otherwise, suggests the presence of a more fundamental error (possibly the same as for BLYP and PBE) that is partly compensated by this weaker HB.

The values for the corresponding diffusivities are shown in Table II The table also shows the effective temperatures ${ }^{20}$ for the different simulations, i.e., the temperature at which the diffusivity of the real liquid equals the AIMD value. The table shows the effective temperature with respect to both normal and heavy water. In Ref. 20 there was no need to refer to the difference between heavy and light water, since the differences in diffusivity between the two $(\sim 25 \%)$ are negligible when compared with the deviation of AIMD (BLYP) with respect to experiment.20 In this study, however, the RPBE description is much closer to experiment, and the distinction becomes relevant.

As shown in Ref. 20, several results for the room-temperature simulations fairly reproduce results of experiments or empirical models at the effective temperature. Besides the diffusivity, features of the RDFs like the heights of the first minimum and the second maximum of the $\mathrm{O}-\mathrm{O}$ RDF would conform to this temperature scaling. The HB network also follows this scaling. In Fig. 2 the distribution of molecular coordinations is compared for RPBE and TIP5P at room temperature and BLYP at $345 \mathrm{~K}$. The RPBE histogram shows a very slightly more over-coordinated liquid than the TIP5P one, suggesting a slightly lower effective temperature than $300 \mathrm{~K}$. A remarkable agreement appears when comparing the diffusivities of both simulations, BLYP at $345 \mathrm{~K}$ and RPBE at $300 \mathrm{~K}$. As reported in Ref. 20 the diffusivity of the former is $\sim 1.5 \times 10^{-5} \mathrm{~cm}^{2} / \mathrm{s}$, identical to the one found for the latter in this work.

Fig. 3] shows the evolution of the diffusivity and relates it to the evolution of the liquid structure, 
TABLE II: Diffusivity $(D)$ obtained in simulations of heavy water for different functionals and in experiment, all at room temperature. Corresponding effective temperatures with respect to light water $\left(T_{e f f}^{\mathrm{H}_{2} \mathrm{O}}\right)$ and heavy water $\left(T_{\text {eff }}^{\mathrm{D}_{2} \mathrm{O}}\right)$.

\begin{tabular}{lcll}
\hline \hline & $D\left(\times 10^{-5} \mathrm{~cm}^{2} / \mathrm{s}\right)$ & $T_{\text {eff }}^{\mathrm{H}_{2} \mathrm{O}}(\mathrm{K})$ & $T_{\text {eff }}^{\mathrm{D}_{2} \mathrm{O}}(\mathrm{K})$ \\
\hline $\mathrm{BLYP}^{a}$ & 0.20 & $240(-20 \%)$ & $250(-17 \%)$ \\
$\mathrm{BLYP}^{a}$ & 0.15 & $237(-21 \%)$ & $245(-18 \%)$ \\
$\mathrm{PBE}^{a}$ & 0.16 & $238(-21 \%)$ & $246(-18 \%)$ \\
$\mathrm{RPBE}^{\mathrm{Exp}}\left(\mathrm{D}_{2} \mathrm{O}\right)^{b}$ & 1.50 & $283(-6 \%)$ & $292(-3 \%)$ \\
$\operatorname{Exp}\left(\mathrm{H}_{2} \mathrm{O}\right)^{b}$ & 1.87 & & \\
\hline \hline
\end{tabular}

${ }^{a}$ Ref. 14; ${ }^{b}$ Ref. 17;

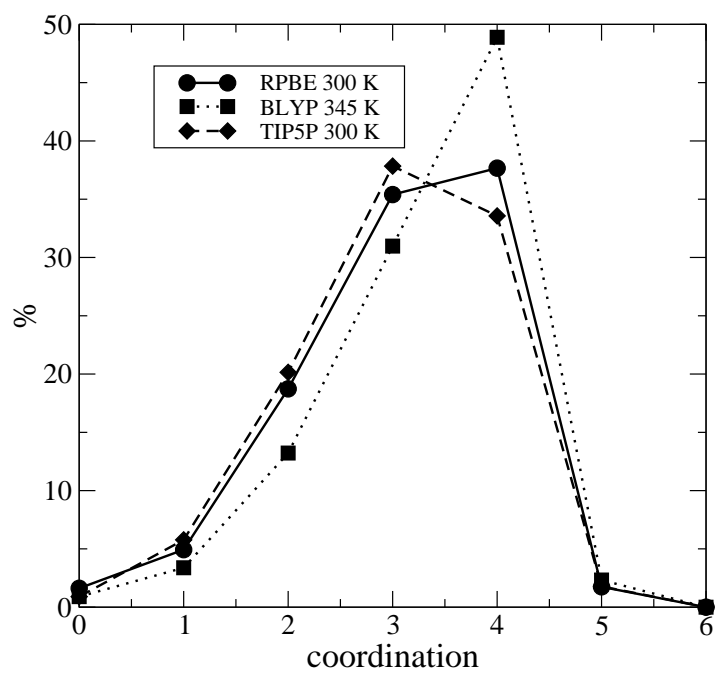

FIG. 2: Distribution of molecules with different coordinations. Room-temperature RPBE (circles, solid line) is compared with the TIP5P potential ${ }^{46}$ (diamonds, dashed line) at room temperature and with BLYP (squares, dotted line) at $345 \mathrm{~K}$.

as monitored by the heights of the first minimum and of the second maximum of $g_{\mathrm{OO}}(r)$. The "moving-window" approach defined in Ref. 20 has been used to compute the time averages. In the RPBE simulation windows of 5 ps were considered every 2 ps, whereas in the BLYP simulation the windows were of 7.5 ps every $2.5 \mathrm{ps}$, given the faster evolution of the former. Even if none of the trajectories has strictly evolved into a stationary situation, the figure reveals that the characteristic time of RPBE is shorter than the one of BLYP. However, from the tentative assimilation of the experimentally measured relaxation times ${ }^{51}$ to our equilibration times, one would expect for an effective temperature of $292 \mathrm{~K}$ a substantially shorted relaxation time. Surprisingly, it does not seem to be the case here, our equilibration time scale being rather on the 10 ps range or longer.

\section{CONCLUSIONS}

We have performed DFT-based AIMD simulations of room-temperature liquid water, comparing the characteristics of the fluid obtained by BLYP and RPBE. The main conclusions are summarised as follows.

(i) The RDFs for RPBE are closer to experiment than the corresponding ones for BLYP. RPBE 


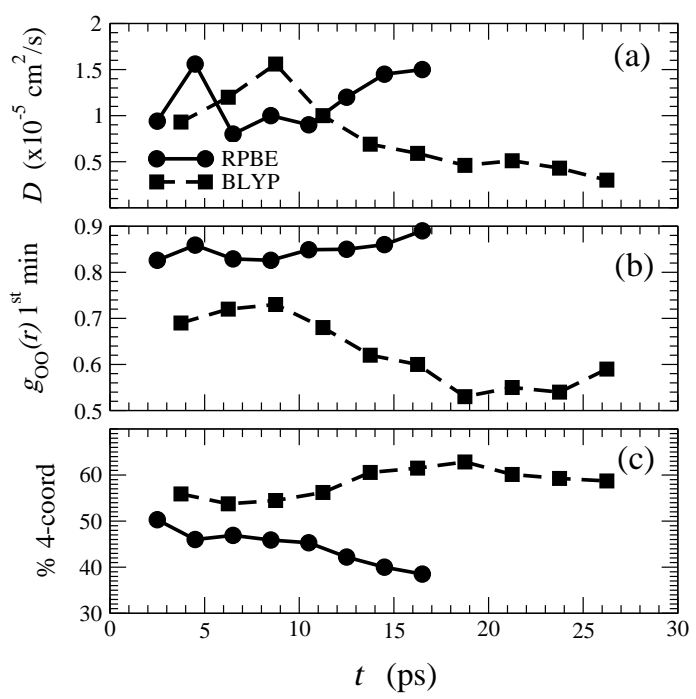

FIG. 3: Evolution of the diffusivity (a), the height of the first minimum of $g_{\mathrm{OO}}(r)$ (b), and the concentration of coordination defects (c), in simulation 3 (RPBE) calculated in 5-ps-windows every 2 ps (solid line, circles) and in simulation 2 (BLYP) calculated in 7.5-ps-windows every 2.5 ps (dashed line, squares).

water is less structured than BLYP, still slightly over-structured with respect to experiment.

(ii) The diffusivity obtained for RPBE is still too low, but much closer to experiment than the value obtained for BLYP, with a factor of two underestimation instead of one order of magnitude.

(iii) For room-temperature AIMD, the effective temperature (with respect to heavy water experimental results) is $\sim 292 \mathrm{~K}(-3 \%)$ for RPBE, while for BLYP it is $\sim 250 \mathrm{~K}(-17 \%)$. In addition to the quantitative advantage of performing RPBE simulations there is a qualitative advantage in that neither of these RPBE simulations would be for supercooled water.

(iv) The AIMD HB-network at AIMD temperature is extremely similar to the TIP5P network at the effective temperature.

$(v)$ The equilibration time-scale is around 10 ps or longer for room-temperature RPBE, as compared with more than 20 ps for BLYP. The fact that it is not substantially shorter is somehow surprising. 20

Even if there are reasons to believe that the good results found for RPBE are not due to fundamental reasons, but rather to error cancellations, still, the possibility of having a well-behaved GGA water is appealing. We consider that it is worth further exploring the performance of RPBE in other wet systems, possibly starting from well characterised ion hydration shells.

\section{Acknowledgments}

We thank M. Sprik and J. VandeVondele for useful discussions. We acknowledge financial support from the British Engineering and Physical Sciences Research Council, the Cambridge European Trust, and the Comunidad Autónoma de Madrid. The calculations were performed in the Cambridge Cranfield High Performance Computing Facility.

* Electronic address: mfer01@esc.cam.ac.uk 
1 Chapman, C., Emmerich, W., Wakelin, J., Artacho, E., Dove, M., and Bruin, R. to be published in the same issue.

2 Litzkow, M., Livny, M., and Mutka, M. Condor - a hunter of idle workstations. In Proceedings of the 8th International Conference of Distributed Computing Systems, , June (1988).

3 García, A., Murray-Rust, P., and Wakelin, J. The use of xml and cml in computational chemistry and physics programs. In Proceeding of the UK e-Science All Hands Meeting, (2004).

4 Wakelin, J., Murray-Rust, P., Tyrrell, S., Zhang, Y., Rezpa, H. S., and García, A. to be published in the same issue.

5 Foster, I. and Kesselman, C., editors. The Grid: Blueprint for a New Computing Infrastructure. Morgan Kaufmann, San Francisco, Calif., (1999).

6 Chapman, C., Emmerich, W., Wakelin, J., Artacho, E., Dove, M., and Bruin, R. to be published in the same issue.

7 Sprik, M., Hutter, J., and Parrinello, M. Ab initio molecular dynamics simulation of liquid water: Comparison of three gradient-corrected density functionals. J. Chem. Phys. 105, 1142 (1996).

8 Ortega, J., Lewis, J. P., and Sankey, O. First principles simulations of fluid water: The radial distribution functions. J. Chem. Phys. 106, 3696 (1996).

9 Silvestrelli, P. L. and Parrinello, M. Structural, electronic, and bonding properties of liquid water from first principles. J. Chem. Phys. 111, 3572 (1999).

10 Silvestrelli, P. L. and Parrinello, M. Water Molecule Dipole in the Gas and in the Liquid Phase. Phys. Rev. Lett. 82, 3308 (1999).

11 Geissler, P. L., Dellago, C., Chandler, D., Hutter, J., and Parrinello, M. Autodissociation of a water molecule in liquid water. Science 291, 2121 (2001).

12 Boero, M., Parrinello, M., Terakura, K., Ikeshoji, T., and Liew, C. C. First-Principles MolecularDynamics Simulations of a Hydrated Electron in Normal and Supercritical Water. Phys. Rev. Lett. 90, $226403(2003)$.

13 Izvekov, S. and Voth, G. A. Car-Parrinello molecular dynamics simulation of liquid water: New results. J. Chem. Phys. 116, 10372 (2002).

14 Grossman, J. C., Schwegler, E., Draeger, E. W., Gygi, F., and Galli, G. Towards an assessment of the accuracy of density functional theory for first principles simulations of water. J. Chem. Phys. 120, 300 (2004).

15 Schwegler, E., Grossman, J. C., Gygi, F., and Galli, G. Towards an assessment of the accuracy of density functional theory for first principles simulations of water II. arXiv:cond-mat, 0405561 (2004).

16 Asthagiri, D., Pratt, L. R., and Kress, J. D. Free energy of liquid water on the basis of quasichemical theory and ab initio molecular dynamics. Phys. Rev. E 68, 41505 (2003).

17 Mills, R. Self-diffusion in normal and heavy water in the range $1-45^{\circ} \mathrm{C}$. J. Chem. Phys. 77, 685 (1973).

18 Soper, A. K. The radial distribution functions of water and ice from $220 \mathrm{~K}$ to $673 \mathrm{~K}$ and at pressures up to 400 MPa. Chem. Phys. 258, 121 (2000).

19 Sorenson, J. M., Hura, G., Glaeser, R. M., and Head-Gordon, T. What can x-ray scattering tell us about the radial distribution functions of water? J. Chem. Phys. 113, 9149 (2000).

20 Fernández-Serra, M. V. and Artacho, E. to be published, preprint in arXiv:cond-mat/0407237 (2004).

21 Giovambattista, N., Starr, F. W., Sciortino, F., Buldyrev, S. V., and Stanley, H. E. Transitions between inherent structures in water. Phys. Rev. E 65(4), 041502 (2002).

22 Becke, A. D. Density-functional exchange-energy approximation with correct asymptotic behavior. Phys. Rev. A 38, 3098 (1998).

23 Lee, C., Yang, W., and Parr, R. G. Development of the Colle-Salvetti correlation-energy formula into a functional of the electron density. Phys. Rev. B 37, 785 (1988).

24 Perdew, J. P., Burke, K., and Ernzerhof, M. Generalized Gradient Approximation Made Simple. Phys. Rev. Lett. 77, 3865 (1996).

25 VandeVondele, J., Mohamed, F., Krack, M., Hutter, J., Sprik, M., and Parrinello, M. to be published.

26 Hammer, B., Hansen, L. B., and Norskov, J. K. Improved adsorption energetics within density-functional theory using revised Perdew-Burke-Ernzerhof functionals. Phys. Rev. B 59, 7413 (1999).

27 Eichler, A. and Hafner, J. NO Reduction over Pt(100): reaction rates from first principles. Chem. Phys. Lett. 343, 383 (2001).

28 Molina, L. M. and Hammer, B. Active Role of Oxide Support during CO Oxidation at Au/MgO. Phys. Rev. Lett. 90, 206102 (2003). 
29 Perdew, J. P. Accurate Density Functional for the Energy: Real-Space Cutoff of the Gradient Expansion for the Exchange Hole. Phys. Rev. Lett. 55, 1665 (1985).

30 Perdew, J. P., Kurth, S., Zupan, A., and Blaha, P. Accurate Density Functional with Correct Formal Properties: A Step Beyond the Generalized Gradient Approximation. Phys. Rev. Lett. 82, 2544 (1999).

31 Becke, A. D. Density-functional thermochemistry. III. The role of exact exchange. J. Chem. Phys. 98, 5648 (1993).

32 Becke, A. D. Density-functional thermochemistry. IV. A new dynamical correlation functional and implications for exact-exchange mixing. J. Chem. Phys. 104, 1040 (1996).

33 Becke, A. D. Density-functional thermochemistry. V. Systematic optimization of exchange-correlation functionals. J. Chem. Phys. 107, 8554 (1997).

34 Kohn, W., Meir, Y., and Makarov, D. E. van der Waals Energies in Density Functional Theory. Phys. Rev. Lett. 80, 4153 (1998).

35 Dion, M., Rydberg, H., Schrder, E., Langreth, D. C., and Lundqvist, B. I. Van der Waals Density Functional for General Geometries. Phys. Rev. Lett. 92, 246401 (2004).

36 Zhang, Y. and Yang, W. Comment on "Generalized Gradient Approximation Made Simple". Phys. Rev. Lett. 80, 890 (1988).

37 Kohn, W. and Sham, L. J. Self-Consistent Equations Including Exchange and Correlation Effects. Phys. Rev. 140, a1133 (1965).

38 Hohenberg, P. and Kohn, W. Inhomogeneous Electron Gas. Phys. Rev. 136, b864 (1964).

39 Troullier, N. and Martins, J. L. Efficient pseudopotentials for plane-wave calculations. Phys. Rev. B 43, 1993 (1991).

40 Kleinman, L. and Bylander, D. M. Efficacious Form for Model Pseudopotentials. Phys. Rev. Lett. 48, 1425 (1982).

41 Ordejón, P., Artacho, E., and Soler, J. M. Self-consistent order-N density-functional calculations for very large systems. Phys. Rev. B 53, 10441 (1996).

42 Soler, J. M., Artacho, E., Gale, J. D., García, A., Junquera, J., Ordejón, P., and Sánchez-Portal, D. The Siesta method for ab initio order-N materials simulation. J Phys. Condens. Matter 14, 2745 (2002).

43 Junquera, J., Paz, O., Sánchez-Portal, D., and Artacho, E. Numerical atomic orbitals for linear-scaling calculations. Phys. Rev. B 64, 235111 (2001).

44 Anglada, E., Soler, J. M., Junquera, J., and Artacho, E. Systematic generation of finite-range atomic basis sets for linear-scaling calculations. Phys. Rev. B 66, 205101 (2002).

45 Allen, M. P. and Tildesley, D. J. "Computer simulation of Liquids". Oxford University Press, (1987).

46 Mahoney, M. W. and Jorgensen, W. L. A five-site model for liquid water and the reproduction of the density anomoly by rigid, nonpolarizable potential functions. J. Chem. Phys. 112, 8910 (2000).

47 Berendsen, H. J. C., Grigera, J. R., and Straatsma, T. P. The Missing Term in Effective Pair Potentials. J. Chem. Phys. 91, 6269 (1987).

48 Berendsen, H.J.C., van der Spoel, D., and van Drunen, R. GROMACS: A message-passing parallel molecular dynamics implementation. Comp. Phys. Comm. 91, 43 (1995).

49 Lindahl, E., Hess, B., and van der Spoel, D. GROMACS 3.0: A package for molecular simulation and trajectory analysis. J. Mol. Mod. 7, 306 (2001).

50 Berendsen, H. J. C., Pstman, J. P. M., van Gunsteren, W. F., DiNola, A., and Haak, J. R. Molecular dynamics with coupling to an external bath. J. Chem. Phys. 81, 3684 (1984).

51 Masciovecchio, C., Santucci, S. C., Gessini, A., Fonzo, S. D., Ruocco, G., and Sette, F. Structural Relaxation in Liquid Water by Inelastic UV Scattering. Phys. Rev. Lett. 92, 255507 (2004). 